\title{
Polytope Contractions within Weyl Group Symmetries
}

\author{
Marzena Szajewska ${ }^{1,2}$
}

Received: 16 February 2016 / Accepted: 4 July 2016 / Published online: 16 July 2016 (C) The Author(s) 2016. This article is published with open access at Springerlink.com

\begin{abstract}
A general scheme for constructing polytopes is implemented here specifically for the classes of the most important 3D polytopes, namely those whose vertices are labeled by integers relative to a particular basis, here called the $\omega$-basis. The actual number of non-isomorphic polytopes of the same group has no limit. To put practical bounds on the number of polytopes to consider for each group we limit our consideration to polytopes with dominant point (vertex) that contains only nonnegative integers in $\omega$-basis. A natural place to start the consideration of polytopes from is the generic dominant weight which were all three coordinates are the lowest positive integer numbers. Contraction is a continuous change of one or several coordinates to zero.
\end{abstract}

Keywords Coxeter group $\cdot$ Reflection group $\cdot 3 \mathrm{D}$ polytopes $\cdot$ Contractions of polytopes $\cdot$ Branching rules

Mathematics Subject Classfication (2010) $20 \mathrm{~F} 55 \cdot 51 \mathrm{~F} 15 \cdot 52 \mathrm{~B} 10 \cdot 65 \mathrm{~N} 45$

Marzena Szajewska

m.szajewska@math.uwb.edu.pl

1 Institute of Mathematics, University of Bialystok, 1M Ciolkowskiego, PL-15-245, Bialystok, Poland

2 Centre de recherches mathématiques, Université de Montréal, C. P. 6128, Centre-ville, Montréal, H3C 3J7, Québec, Canada 


\section{Introduction}

Contractions of reflection generated polytopes in $3 \mathrm{D}$ real Euclidean space $\mathbb{R}^{3}$ were introduced in [2]. Although the method is quite general, in terms of the dimension of the space and the reflection group symmetry, it has been used specifically for polytopes with icosahedral symmetry only [2]. More precisely, a polytope is being deformed into another polytope by the contraction process, while the icosahedral symmetry is preserved at all stages of the process.

In this paper we consider contractions of the polytopes with symmetries that include the Weyl reflection groups associated with the simple Lie algebras of rank 3 $[5,6,10]$. Those are the Lie algebras of type $A_{3}, B_{3}$, and $C_{3}$. The Weyl group of $A_{3}$ is isomorphic to the symmetry group of 4 elements, and is thus of order 24 . The Weyl groups of $B_{3}$ and $C_{3}$ are isomorphic to each other. Therefore their polytopes have a certain resemblance, for example as a cube and regular octahedron. Nevertheless their contractions are sufficiently different.

A motivation for the polytope contractions can be found in [1, 2, 9]. The analogy with the definition of contractions of Lie algebras established some 60 years ago [7], is in itself a motivation for our present undertaking.

Sooner or later one may envisage the use of the following property of polytopes, which is a direct consequence of their contractions. A polytope whose vertices are situated on a sphere of a certain radius, say $R$, is diminished during the contraction. In order to keep the radius unchanged, coordinates of the remaining vertices have to be correspondingly increased. During that process the overall symmetry of the polytope does not change. If we let the coordinates of one vertex vibrate, then the coordinates of the rest of the vertices have to vibrate in opposite phase to preserve the radius of the polytope [3].

\section{Preliminary}

In this section we recall certain facts about the symmetry groups considered in this paper.

\section{$2.1 W\left(A_{3}\right)$ Symmetry Group}

The symmetry group of $W\left(A_{3}\right)$ is the Weyl group of the simple Lie algebra $A_{3}$. Its order is $4 !=24$. It is known to be isomorphic to the permutation group of four elements. It is generated by the reflections $r_{1}, r_{2}, r_{3}$ in the mirrors $m_{1}, m_{2}, m_{3}$, intersecting at the origin of $\mathbb{R}^{3}$ of the real Euclidean space of dimension 3 (see for example $[6,10])$. More details about 3D polytopes of this kind are found in Table 2 in [4].

The simple roots $\alpha_{1}, \alpha_{2}, \alpha_{3}$ are the normal vectors to the three mirrors. It is convenient to show the simple roots as nodes of a Coxeter diagram (see Fig. 1). Relative 


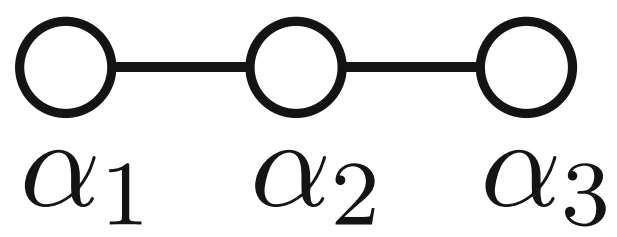

Fig. 1 Coxeter diagram of simple roots for $A_{3}$. Nodes describe simple roots and a link between them indicates an angle between vectors. An angle is equal $\frac{2 \pi}{3}$. If there is no link between nodes it means that the angle is $\frac{\pi}{2}$

angles of the mirrors are also conveniently specified in the case of $W\left(A_{3}\right)$ in terms of the matrix of scalar products of simple roots (the Cartan matrix).

$$
C=\left(\left\langle\alpha_{j}, \alpha_{k}\right\rangle\right)=\left(\begin{array}{ccc}
2 & -1 & 0 \\
-1 & 2 & -1 \\
0 & -1 & 2
\end{array}\right) \text {. }
$$

The simple roots are normalized to $\sqrt{2}$. The normalization is a matter of convenience which makes the Cartan matrix contain the smallest integers as matrix elements.

In the paper we also make extensive use of the dual basis which is the reciprocal of the $\alpha$-basis. It is defined by

$$
\left\langle\alpha_{j}, \omega_{k}\right\rangle=\frac{\left\langle\alpha_{j}, \alpha_{k}\right\rangle}{2} \delta_{j k}, \quad j, k=1,2,3 .
$$

Thus we have [4]

$$
\begin{gathered}
\alpha_{j}=\sum_{k=1}^{3}\left\langle\alpha_{j}, \alpha_{k}\right\rangle \omega_{k}, \quad \omega_{k}=\sum_{j=1}^{3}\left\langle\omega_{k}, \omega_{j}\right\rangle \alpha_{j}, \\
\left(\left\langle\omega_{i}, \omega_{j}\right\rangle\right)=\frac{1}{4}\left(\begin{array}{lll}
3 & 2 & 1 \\
2 & 4 & 2 \\
1 & 2 & 3
\end{array}\right)
\end{gathered}
$$

The vectors of the $\omega$-basis are not of the same length, $\left\langle\omega_{1}, \omega_{1}\right\rangle \neq\left\langle\omega_{2}, \omega_{2}\right\rangle \neq$ $\left\langle\omega_{3}, \omega_{3}\right\rangle$. The reflections of $W\left(A_{3}\right) r_{1}, r_{2}, r_{3}$ in the mirrors $m_{1}, m_{2}, m_{3}$ respectively, transform a vector $(x, y, z) \in \mathbb{R}^{3}$ given in the $\omega$-basis, as follows,

$$
\begin{aligned}
& r_{1}(x, y, z)=(-x, x+y, z), \\
& r_{2}(x, y, z)=(x+y,-y, y+z), \\
& r_{3}(x, y, z)=(x, y+z,-z) .
\end{aligned}
$$

\section{$2.2 W\left(B_{3}\right)$ Symmetry Group}

The symmetry group $W\left(B_{3}\right)$ is generated by the reflections $r_{1}, r_{2}, r_{3}$ in mirrors intersecting at the origin of $\mathbb{R}^{3}$ defined by their normal vectors $\alpha_{1}, \alpha_{2}, \alpha_{3}$. The Coxeter diagram for $B_{3}$ is shown in Fig. 2 . 


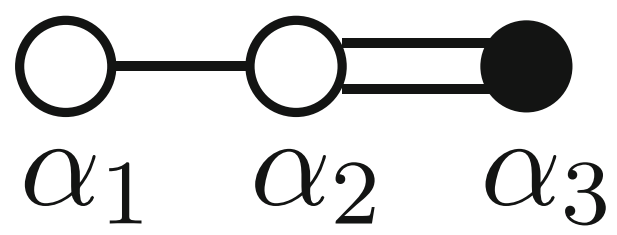

Fig. 2 Coxeter diagram of simple roots for $B_{3}$. Nodes describe simple roots and a link between them indicates an angle between vectors. One of the angle is equal to $\frac{2 \pi}{3}$. If there is no link between nodes it means that the angle is $\frac{\pi}{2}$. A double line describes the angle $\frac{3 \pi}{4}$. A black node means a shorter root vector

The relative position of the mirrors is given by the relative position of their normals. The Cartan matrix of $B_{3}$ differs from that of $A_{3}(2.1)$.

$$
C=\left(\begin{array}{ccc}
2 & -1 & 0 \\
-1 & 2 & -2 \\
0 & -1 & 2
\end{array}\right), \quad\left(\left\langle\omega_{j}, \omega_{k}\right\rangle\right)=\frac{1}{2}\left(\begin{array}{ccc}
2 & 2 & 1 \\
2 & 4 & 2 \\
1 & 2 & 3 / 2
\end{array}\right)
$$

The simple roots are of different length and are normalized to $\sqrt{2}$ and 1 .

$$
\left|\alpha_{1}\right|=\left|\alpha_{2}\right|=\sqrt{2}, \quad\left|\alpha_{3}\right|=1 .
$$

The reflections of $W\left(B_{3}\right) r_{1}, r_{2}, r_{3}$ in mirrors $m_{1}, m_{2}, m_{3}$ respectively, transform a vector $(x, y, z) \in \mathbb{R}^{3}$ given in the $\omega$-basis, as follows,

$$
\begin{aligned}
& r_{1}(x, y, z)=(-x, x+y, z), \\
& r_{2}(x, y, z)=(x+y,-y, 2 y+z), \\
& r_{3}(x, y, z)=(x, y+z,-z) .
\end{aligned}
$$

\section{$2.3 W\left(C_{3}\right)$ Symmetry Group}

As in the previous subsections the symmetry group $W\left(C_{3}\right)$ is generated by the reflections $r_{1}, r_{2}, r_{3}$ in mirrors intersecting at the origin of $\mathbb{R}^{3}$ defined by their normal vectors $\alpha_{1}, \alpha_{2}, \alpha_{3}$. The Coxeter diagram for $C_{3}$ is shown in Fig. 3.

The Cartan matrix of $C_{3}$ has the following form:

$$
C=\left(\begin{array}{ccc}
2 & -1 & 0 \\
-1 & 2 & -1 \\
0 & -2 & 2
\end{array}\right), \quad\left(\left\langle\omega_{j}, \omega_{k}\right\rangle\right)=\frac{1}{2}\left(\begin{array}{lll}
1 & 1 & 1 \\
1 & 2 & 2 \\
1 & 2 & 3
\end{array}\right)
$$

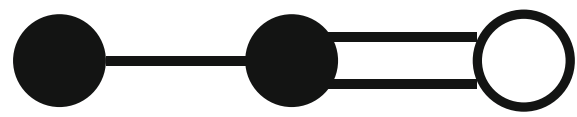

Fig. 3 Coxeter diagram of simple roots for $C_{3}$. Nodes describe simple roots and a link between them indicates an angle between vectors. One of the angle is equal to $\frac{2 \pi}{3}$. If there is no link between nodes it means that the angle is $\frac{\pi}{2}$. A double line describes the angle $\frac{3 \pi}{4}$. Black nodes describes shorter root vectors 
The simple roots, as for the $B_{3}$ case, are of different length and are normalized to $\sqrt{2}$ and 1.

$$
\left|\alpha_{1}\right|=\left|\alpha_{2}\right|=1, \quad\left|\alpha_{3}\right|=\sqrt{2} .
$$

The difference between $B_{3}$ and $C_{3}$ is that the long roots of $B_{3}$ are the short roots of $C_{3}$ and short root of $B_{3}$ is a long root of $C_{3}$. The reflections of the $W\left(C_{3}\right) r_{1}, r_{2}, r_{3}$ in the mirrors $m_{1}, m_{2}, m_{3}$ respectively, transform a vector $(x, y, z) \in \mathbb{R}^{3}$ given in the $\omega$-basis, as follows,

$$
\begin{aligned}
& r_{1}(x, y, z)=(-x, x+y, z), \\
& r_{2}(x, y, z)=(x+y,-y, y+z), \\
& r_{3}(x, y, z)=(x, y+2 z,-z) .
\end{aligned}
$$

For any of the three groups we can choose a point with coordinates $(a, b, c)$ in the $\omega$-basis. Applying to it reflections of its Weyl group in every way as long as distinct points are created we form an orbit of the Weyl group. The points of such an orbit are interpreted as vertices of a polytope in $\mathbb{R}^{3}$. Its vertices are equidistant from the origin. The number of points belong to the orbits of each Weyl group are summarized in Table 1.

\section{The Family of $W\left(A_{3}\right), W\left(B_{3}\right)$ and $W\left(C_{3}\right)$ Polytopes}

Any orbit of the symmetry group is specified by its unique point, called the dominant point, which has non-negative coordinates in the $\omega$-basis. The orbit of the point $(0,0,0)$ is an origin of the space. The dominant weight can take real non-negative values of its coordinates in the $\omega$-basis and 2 different choices of these coordinates lead to different orbits even if the group is the same for all the cases. The vertices of polytopes are generated by reflections starting from the dominant point $(a, b, c)$, where $a, b, c \geq 0$. For each group such points are listed in the Appendix.

The largest orbit $(a, b, c)$, where $a, b, c>0$, has 24, 48 and 48 distinct points for $A_{3}, B_{3}$ and $C_{3}$, respectively. For the positive parameters $a, b, c$ there are 3 edges meeting at each vertex $P$. The edges are formed by reflecting the vertex $P$ in 3 directions given by simple roots, namely $\left[P, r_{1} P\right],\left[P, r_{2} P\right],\left[P, r_{3} P\right]$.

Table 1 Orbit sizes for arbitrary points $(a, b, c)$, where $a, b, c>0$ for each group

\begin{tabular}{llll}
\hline Orbit size & $A_{3}$ & $B_{3}$ & $C_{3}$ \\
\hline$\left|W_{(a, b, c)}\right|$ & 24 & 48 & 48 \\
$\left|W_{(a, b, 0)}\right|$ & 12 & 24 & 24 \\
$\left|W_{(a, 0, c)}\right|$ & 12 & 24 & 24 \\
$\left|W_{(0, b, c)}\right|$ & 12 & 24 & 24 \\
$\left|W_{(0, b, 0)}\right|$ & & & \\
$\left|W_{(0,0, c)}\right|$ & 6 & 12 & 12 \\
$\left|W_{(a, 0,0)}\right|$ & 4 & 8 & 6 \\
$\left|W_{(0,0,0)}\right|$ & 4 & 6 & 1 \\
\hline
\end{tabular}




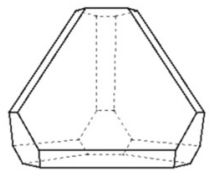

$(1 / 6,1 / 3,1)$

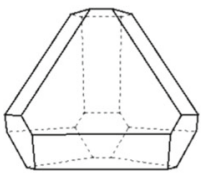

$(1 / 3,1 / 6,1)$

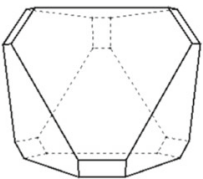

$(1 / 6,1,1 / 3)$

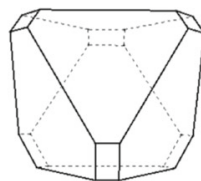

$(1 / 3,1,1 / 6)$

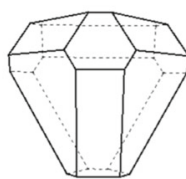

$(1,1 / 6,1 / 3)$

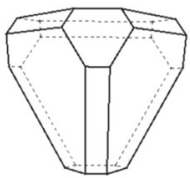

$(1,1 / 3,1 / 6)$

Fig. 4 Examples of polytopes with tetrahedral $W\left(A_{3}\right)$ symmetry. Each of the polytopes has 24 vertices. There are 2 different orbits of edges, namely, hexagon-rectangular, hexagon-hexagon

The length of the edges meeting at the dominant point $P=a \omega_{1}+b \omega_{2}+c \omega_{3}$ is shown below, where the scalar products of the $\omega$ 's are taken from (2.2), (2.4), (2.6), respectively for each type of polytope.

$\left|\left[P, r_{1} P\right]\right|=\sqrt{\left\langle a \omega_{1}+b \omega_{2}+c \omega_{3}-r_{1}\left(a \omega_{1}+b \omega_{2}+c \omega_{3}\right)\right\rangle^{2}}=a \sqrt{2}$ for each case ,

$\left|\left[P, r_{2} P\right]\right|=\sqrt{\left\langle a \omega_{1}+b \omega_{2}+c \omega_{3}-r_{2}\left(a \omega_{1}+b \omega_{2}+c \omega_{3}\right)\right\rangle^{2}}=\left\{\begin{array}{cc}b \sqrt{2} & \text { for } W\left(A_{3}\right) \text { and } W\left(B_{3}\right) \\ b & \text { for } W\left(C_{3}\right) \\ c \sqrt{2} & \text { for } W\left(A_{3}\right) \\ c & \text { for } W\left(B_{3}\right) \text { and } W\left(C_{3}\right)\end{array}\right.$,

We present polytopes which preserve tetrahedral $W\left(A_{3}\right)$ and octahedral $W\left(B_{3}\right), W\left(C_{3}\right)$ symmetries. We choose the three values $1, \frac{1}{3}, \frac{1}{6}$ for the parameters $a, b, c$. The six resulting polytopes are shown in Fig. 4, 5 and 6 .

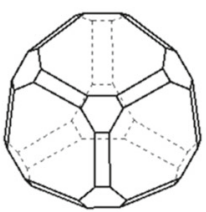

$(1 / 6,1 / 3,1)$

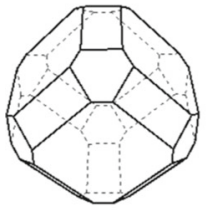

$(1 / 3,1 / 6,1)$

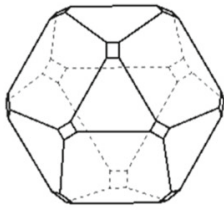

$(1 / 6,1,1 / 3)$

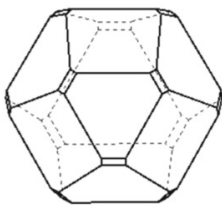

$(1 / 3,1,1 / 6)$

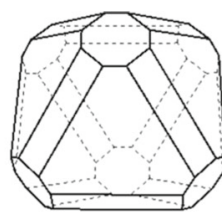

$(1,1 / 6,1 / 3)$

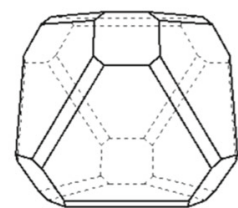

$(1,1 / 3,1 / 6)$

Fig. 5 Examples of polytopes with octahedral $W\left(B_{3}\right)$ symmetry. Each of the polytopes has 48 vertices. There are 3 different orbits of edges, namely, hexagon-rectangular, hexagon-octagon, octagon-rectangular 


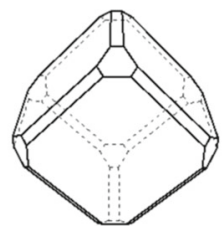

$(1 / 6,1 / 3,1)$

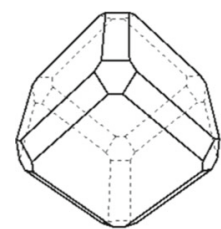

$(1 / 3,1 / 6,1)$

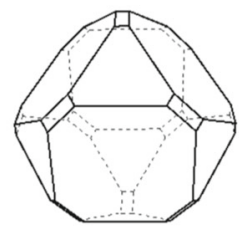

$(1 / 6,1,1 / 3)$

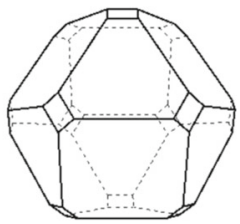

$(1 / 3,1,1 / 6)$

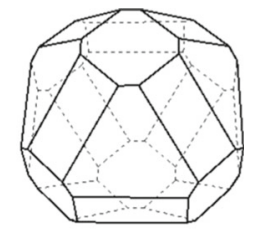

$(1,1 / 6,1 / 3)$

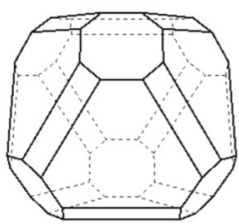

$(1,1 / 3,1 / 6)$

Fig. 6 Examples of polytopes with octahedral $W\left(C_{3}\right)$ symmetry. Each of the polytopes has 48 vertices. There are 3 different orbits of edges, namely, hexagon-rectangular, hexagon-octagon, octagon-rectangular

Looking at Fig. 4 one notices that the polytopes with dominant weight, where the first and third coordinate are interchanged are the same. This is an effect of a symmetry of group $A_{3}$ visible in the Coxeter diagrams in Fig. 1.

The number of edges and 2-faces in each polytope can be read from Table 2.

\section{Contraction of $W\left(A_{3}\right), W\left(B_{3}\right), W\left(C_{3}\right)$ Polytopes}

Most often the polytopes of interest have the seed point $(a, b, c)$ with integer coordinates relative to the $\omega$-basis. However here, during the contraction process [8], the coordinates of the point will be allowed to change continuously, taking positive non-integer values as well. Let us emphasize that at every stage of that continuous contraction process of the polytope, exact symmetry of the polytope is preserved. However the distances between the vertices and the origin vary through the contraction process.

Table 2 Numbers of vertices/edges/2-faces in the polytopes with dominant point $(a, b, c)$, where $a, b, c>0$

\begin{tabular}{llll}
\hline dominant point & $A_{3}$ & $B_{3}$ & $C_{3}$ \\
\hline$(a, b, c)$ & $24 / 36 / 14$ & $48 / 72 / 26$ & $48 / 72 / 26$ \\
$(a, b, 0)$ & $12 / 18 / 8$ & $24 / 36 / 14$ & $24 / 36 / 14$ \\
$(a, 0, c)$ & $12 / 24 / 14$ & $24 / 48 / 26$ & $24 / 48 / 26$ \\
$(0, b, c)$ & $12 / 18 / 8$ & $24 / 36 / 14$ & $24 / 36 / 14$ \\
$(a, 0,0)$ & $4 / 6 / 4$ & $6 / 12 / 8$ & $6 / 12 / 8$ \\
$(0, b, 0)$ & $6 / 12 / 8$ & $12 / 24 / 14$ & $12 / 24 / 14$ \\
$(0,0, c)$ & $4 / 6 / 4$ & $8 / 12 / 6$ & $8 / 12 / 6$ \\
\hline
\end{tabular}




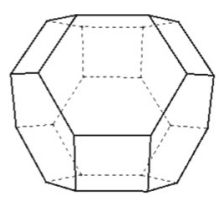

$(1,1,1)$

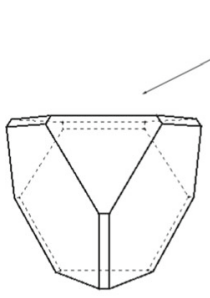

$(1,1,1 / 10)$

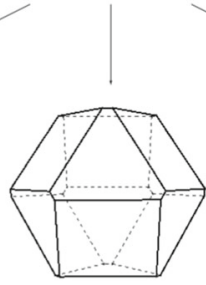

$(1,1 / 10,1)$

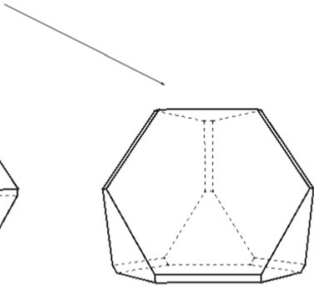

$(1 / 10,1,1)$

Fig. 7 Contractions of the tetrahedral polytope with 24 vertices. On the top row the polytope with dominant point $(1,1,1)$ is shown. On the bottom row the result of the contractions is found for $(1,1,1 / 10)$, $(1,1 / 10,1)$ and $(1 / 10,1,1)$

Definition 4.1 The contraction of any polytope with dominant point $(a, b, c)$ to the polytope with dominant point $\left(a^{\prime}, b^{\prime}, c^{\prime}\right)$ is a deformation of the parameters $a, b, c$ to the parameters $a^{\prime}, b^{\prime}, c^{\prime}$ such that $a \geq a^{\prime} \geq 0, b \geq b^{\prime} \geq 0$ and $c \geq c^{\prime} \geq 0$.

As a starting point for showing the contractions we choose the dominant point $(1,1,1)$. In the first step we change only one of the lengths of the three edges in the polytope. For example we reduce one of the edges to 1/10. Fig. 7, 8 and 9 present the first step of the contraction.

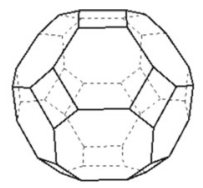

$(1,1,1)$

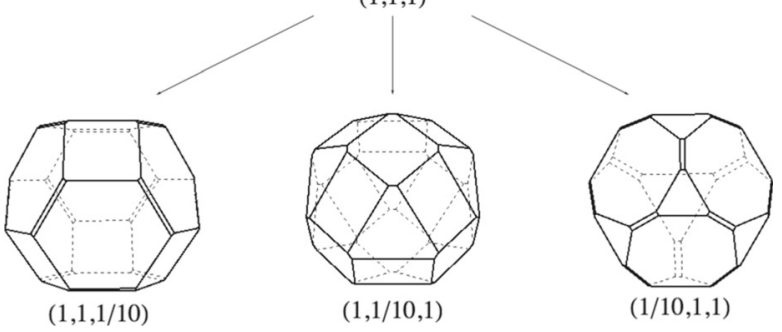

Fig. 8 First step of contractions of the octahedral polytope with 48 vertices for $W\left(B_{3}\right)$. The first row shows the initial polytope before contraction. The second row shows the polytopes with one coordinate changed 


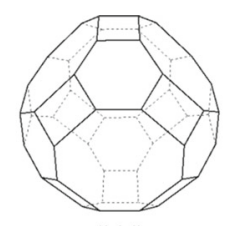

$(1,1,1)$

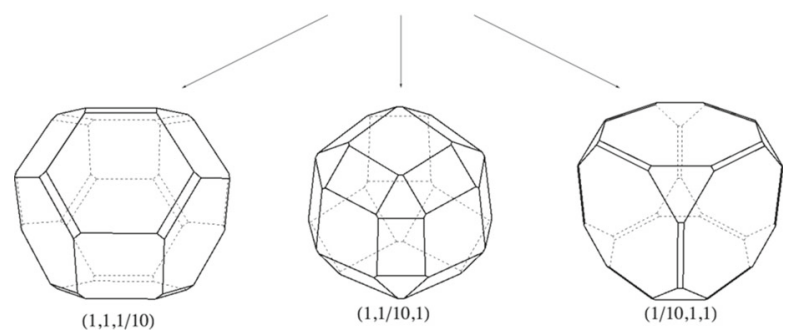

Fig. 9 First step of contractions of the octahedral polytope with 48 vertices for $W\left(C_{3}\right)$. The first row shows the initial polytope before contraction. The second row shows the polytopes with one coordinate changed

\subsection{Contractions of the Polytopes of the Families $(a, b, 0),(0, b, c)$ and $(a, 0, c)$}

In this section we consider polytopes after the first contraction where one of the coordinates in the $\omega$-basis is zero.

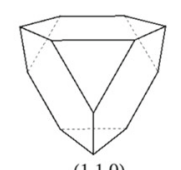

$(1,1,0)$
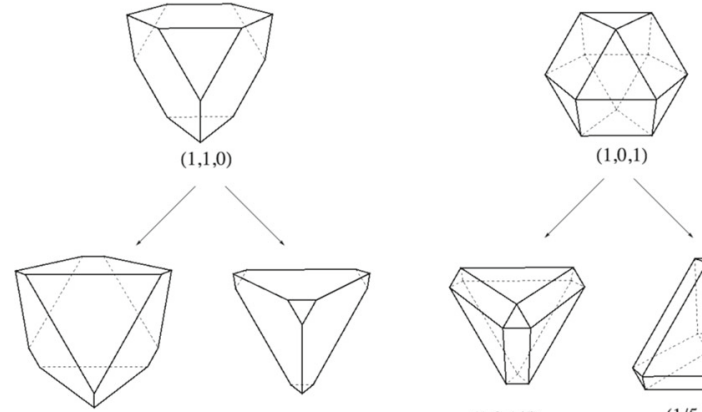

$(1 / 5,1,0)$

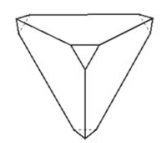

$(1,1 / 5,0)$

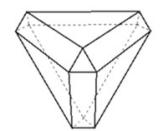

$(1,0,1 / 5)$

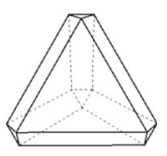

$(1 / 5,0,1)$

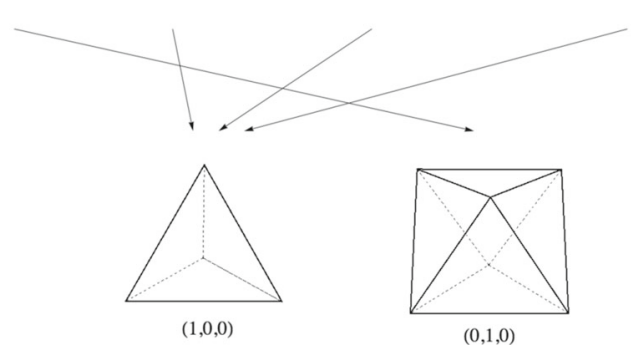

Fig. 10 Contractions of the tetrahedral polytope with 24 vertices. In the top row the two polytopes are shown. In the middle row four polytopes are shown at the stage of contraction where the limit is still not obtained. In the bottom row the end result of the contractions is found. The dominant point $(1,0,0)$ labels the tetrahedron and the point $(0,1,0)$ indicates the octahedron. Arrows show the direction of the contractions 
Transforming only one of the dominant coordinates to zero, one gets polytopes of several kinds. If the dominant point of the polytope has one coordinate equal to zero its properties are quite different (see Table 2). Fig. 10, 11 and 12 show the next step of contractions.

\section{Contractions of Radii of Polytopes}

Since the vertices of a polytope generated by the reflections of the Weyl group are equidistant from the origin, the radii of a polytope with dominant point $(a, b, c)$ can be found by the formula

$$
R=\sqrt{\left(\begin{array}{lll}
a & b & c
\end{array}\right)\left(\left\langle\omega_{i}, \omega_{j}\right\rangle\right)\left(\begin{array}{l}
a \\
b \\
c
\end{array}\right)}
$$

Using the matrices $\left(\left\langle\omega_{i}, \omega_{j}\right\rangle\right)$ from (2.2), (2.4) and (2.6) we have the exact form of the square of the radius of a polytope with dominant point $(a, b, c)$ for each group

$$
R^{2}= \begin{cases}\frac{3}{4} a^{2}+a b+b^{2}+\frac{1}{2} a c+b c+\frac{3}{4} c^{2} & \text { for } A_{3} \\ a^{2}+2 a b+2 b^{2}+a c+2 b c+\frac{3}{4} c^{2} & \text { for } B_{3} \\ \frac{1}{2} a^{2}+a b+b^{2}+a c+2 b c+\frac{3}{2} c^{2} & \text { for } C_{3}\end{cases}
$$

In Table 3 we present some examples of radii for a few dominant points for each group considered in the paper. In Fig. 13 we show how the length of the radii for the polytopes with dominant weights $(1,1,1),(1,1,0)$ and $(1,0,0)$ is changed.

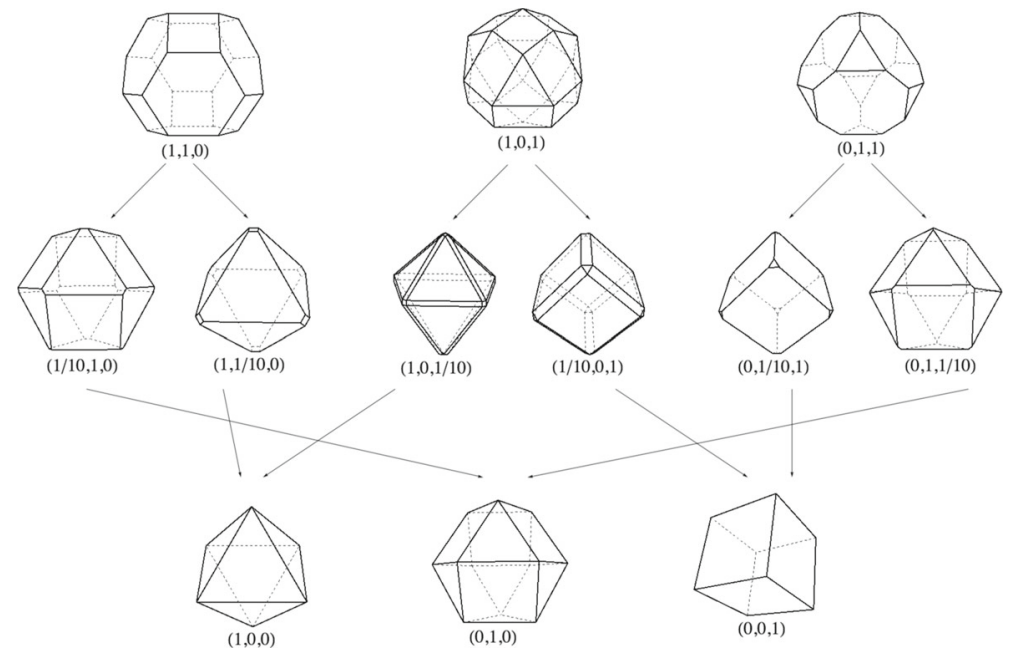

Fig. 11 Contractions of the octahedral polytope with 48 vertices. In the top row the three polytopes are shown. In the bottom row the end result of the contractions is found. The dominant point $(1,0,0)$ labels the octahedron, the point $(0,1,0)$ describes cuboctahedron and the point $(0,1,0)$ indicates the cube. Arrows mark the direction of the contractions 

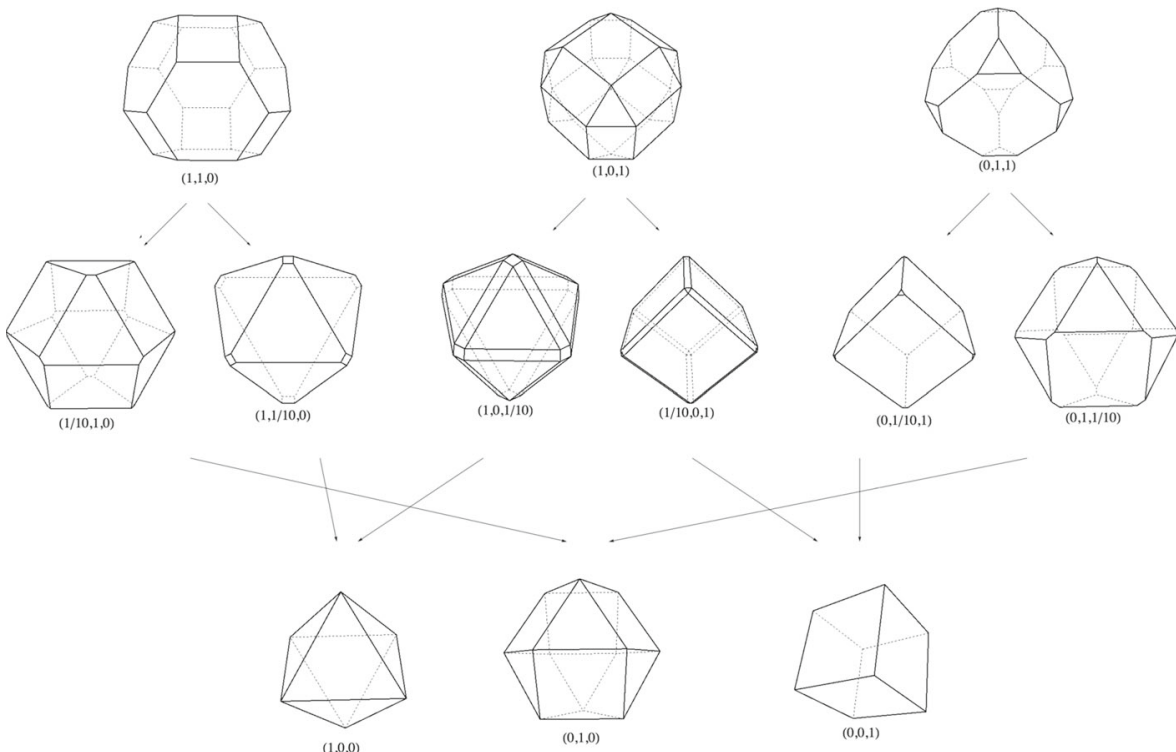

Fig. 12 Contractions of the octahedral polytope with 24 vertices. In the top row the three polytopes are shown. In the bottom row the end result of the contractions is found. The dominant point $(1,0,0)$ labels the octahedron, the point $(0,1,0)$ point describes cuboctahedron and the point $(0,1,0)$ indicates the cube. Arrows show the direction of the contractions

\section{Coalescence of Vertices}

In this section we consider the process when one of the coordinates of the dominant point of the polytope goes to zero. Results of a coalescence of vertices during the contractions are shown for all groups. During the contraction, the number of vertices of a polytope does not change. The vertices just coalesce. The final contraction of a polytope reduces to one point - the origin. This means that all vertices go to the point $(0,0,0)$.

$$
\lim _{a, b, c \rightarrow 0}(a, b, c)=\left|W_{(a, b, c)}\right|(0,0,0) .
$$

Table 3 Values of the square radii of some of the polytopes from each considered group with the given dominant point. The dominant points of the vertices of the polytopes are shown in the $\omega$-basis. The dominant point and the approximate value of the square of its radius are shown in the table

\begin{tabular}{llll}
\hline dominant point & $A_{3}$ & $B_{3}$ & $C_{3}$ \\
\hline$(1,1,1)$ & 5 & 8.75 & 7 \\
$(0,1,1)$ & 2.75 & 4.75 & 4.5 \\
$(1,1,0)$ & 2.75 & 5 & 2.5 \\
$(1,0,1)$ & 2 & 2.75 & 3 \\
$(0,1,0)$ & 1 & 2 & 1 \\
$(0,0,1)$ & 0.75 & 0.75 & 1.5 \\
$(1,0,0)$ & 0.75 & 1 & 0.5
\end{tabular}




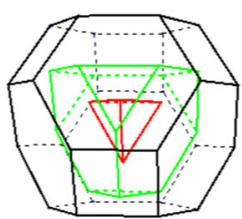

$A_{3}$

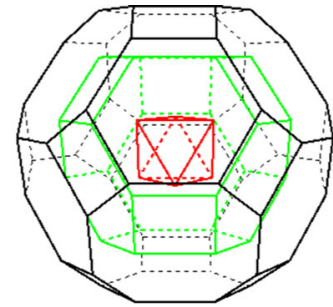

$B_{3}$

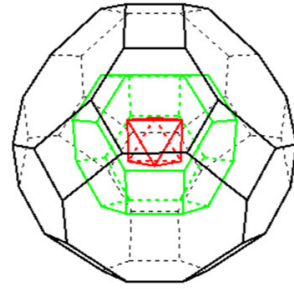

$C_{3}$

Fig. 13 Polytopes with dominant weights $(1,1,1),(1,1,0)$ and $(1,0,0)$ for the Weyl groups $W\left(A_{3}\right), W\left(B_{3}\right), W\left(C_{3}\right)$

Assuming that $a, b, c>0$, the polytope on the left and the polytopes on the right have the same number of vertices. The contraction limits are the following

$$
\begin{aligned}
& \lim _{a \rightarrow 0}(a, b, c)=\frac{\left|W_{(a, b, c)}\right|}{\left|W_{(0, b, c)}\right|}(0, b, c) \\
& \lim _{b \rightarrow 0}(a, b, c)=\frac{\left|W_{(a, b, c)}\right|}{\left|W_{(a, 0, c)}\right|}(a, 0, c) \\
& \lim _{c \rightarrow 0}(a, b, c)=\frac{\left|W_{(a, b, c)}\right|}{\left|W_{(a, b, 0)}\right|}(a, b, 0) .
\end{aligned}
$$

And similarly we have

$$
\begin{array}{ll}
\lim _{a \rightarrow 0}(a, b, 0)=\frac{\left|W_{(a, b, 0)}\right|}{\left|W_{(0, b, 0)}\right|}(0, b, 0) & \lim _{b \rightarrow 0}(a, b, 0)=\frac{\left|W_{(a, b, 0)}\right|}{\left|W_{(a, 0,0)}\right|}(a, 0,0) \\
\lim _{a \rightarrow 0}(a, 0, c)=\frac{\left|W_{(a, 0, c)}\right|}{\left|W_{(0,0, c)}\right|}(0,0, c) & \lim _{c \rightarrow 0}(a, 0, c)=\frac{\left|W_{(a, 0, c)}\right|}{\left|W_{(a, 0,0)}\right|}(a, 0,0) \\
\lim _{b \rightarrow 0}(0, b, c)=\frac{\left|W_{(0, b, c)}\right|}{\left|W_{(0,0, c)}\right|}(0,0, c) & \lim _{c \rightarrow 0}(0, b, c)=\frac{\left|W_{(0, b, c)}\right|}{\left|W_{(0, b, 0)}\right|}(0, b, 0)
\end{array}
$$

We can also combine the contractions (6.1) and (6.2), as for example,

$\lim _{a \rightarrow 0} \lim _{b \rightarrow 0}(a, b, c)=\lim _{a \rightarrow 0} \frac{\left|W_{(a, b, c)}\right|}{\left|W_{(a, 0, c)}\right|}(a, 0, c)=\frac{\left|W_{(a, b, c)}\right|}{\left|W_{(a, 0, c)}\right|} \cdot \frac{\left|W_{(a, 0, c)}\right|}{\left|W_{(0,0, c)}\right|}(0,0, c)=\frac{\left|W_{(a, b, c)}\right|}{\left|W_{(0,0, c)}\right|}(0,0, c)$.

\section{Concluding Remarks and Open Problems}

- There are seven cases of semisimple Lie groups of rank 3. The three which are simple among them have been described in this paper. A description of the four which are semisimple and not simple would also be interesting to consider.

- The polytopes we consider here were summarized in Table 2 by [4]. In that paper corresponding results are found also for polytopes of dimension 4 in Table 3 and 4. 
- During the reduction of the symmetry process the initial group may be reduced to its subgroup. When the two groups coincide we speak about contraction [8] if the second group is smaller we would speak of ,branching rules' [10].

- A chain of polytopes attached to each other by equal sides would be interesting to classify.

- The polytopes with the same symmetry formed by more than one orbit in the outer shell can also be created and would certainly find applications in science.

Acknowledgments The author would like to thank Professor J. Patera (Université de Montréal) for stimulating discussions and comments. The author would like to express her gratitude to the Centre de Recherches Mathématiques, Université de Montréal, for their hospitality during her postdoctoral fellowship. She is also grateful to MITACS for their contribution to her financial support.

Open Access This article is distributed under the terms of the Creative Commons Attribution 4.0 International License (http://creativecommons.org/licenses/by/4.0/), which permits unrestricted use, distribution, and reproduction in any medium, provided you give appropriate credit to the original author(s) and the source, provide a link to the Creative Commons license, and indicate if changes were made.

\section{Appendix}

Exact coordinates of the families of polytopes with dominant point $(a, b, c)$ of the groups $A_{3}, B_{3}$ and $C_{3}$ are shown here. The coordinates $a, b, c$ are given in the $\omega$-basis. The dominant point $(a, b, c)$ is uniquely specified by the three real coordinates, such that $a, b, c>0$. All the vertices are on a sphere of radius equal to the length $|(a, b, c)|$. The points are calculated by applying repeatedly the reflections (2.3), (2.5) and (2.7) to the dominant point $(a, b, c)$ of the groups $A_{3}, B_{3}$ and $C_{3}$, respectively.

For the $A_{3}$ vertices, we have

$$
\begin{array}{llll}
(a, b, c), & (-a, a+b, c), & (-c,-b,-a), & (-c,-a-b, a), \\
(a+b,-b, b+c), & (-b-c, b,-a-b), & (a, b+c,-c), & (c,-b-c,-a), \\
(b,-a-b, a+b+c), & (-a-b-c, a+b,-b), & (-a, a+b+c,-c), \quad(c,-a-b-c, a), \\
(-a-b, a, b+c), & (-b-c,-a, a+b), & (a+b, c,-b-c), \quad(b+c,-c,-a-b), \\
(a+b+c,-b-c, b), & (-b, b+c,-a-b-c), & (-b,-a, a+b+c), \quad(b, c,-a-b-c), \\
(-a-b, a+b+c,-b-c), & (-a-b-c, a, b), & (a+b+c,-c,-b), \quad(b+c,-a-b-c, a+b) .
\end{array}
$$

For the $B_{3}$ vertices, we have

$$
\begin{array}{lll} 
\pm(a, b, c), & \pm(-a, a+b, c), & \pm(b+c,-a-b-c, 2 a+2 b+c), \\
\pm(a, b+c,-c), & \pm(b,-a-b, 2 a+2 b+c), & \pm(-b, a+2 b+c,-2 a-2 b-c), \\
\pm(-a-b, a, 2 b+c), & \pm(a+b, b+c,-2 b-c), & \pm(-a-b, a+2 b+c,-2 b-c), \\
\pm(a+b,-b, 2 b+c), & \pm(-b,-a, 2 a+2 b+c), & \pm(b+c,-a-2 b-c, 2 a+2 b+c), \\
\pm(-a, a+b+c,-c), & \pm(a+2 b+c,-a-b-c, c), & \pm(-a-b-c, a+2 b+c,-2 b-c), \\
\pm(a+b+c, b,-2 b-c), & \pm(a+2 b+c,-b-c, c), & \pm(a+b+c,-b-c, 2 b+c), \\
\pm(-a-b-c, a, 2 b+c), & \pm(-a-2 b-c, a+b, c), & \pm(b+c, a+b,-2 a-2 b-c), \\
\pm(a+2 b+c,-b,-c), & \pm(-b-c,-a, 2 a+2 b+c), & \pm(b, a+b+c,-2 a-2 b-c) .
\end{array}
$$


For the $C_{3}$ vertices, we have

$$
\begin{array}{lll} 
\pm(a, b, c), & \pm(b+2 c, a+b,-a-b-c), & \pm(a+2 b+2 c,-b-2 c, c), \\
\pm(-a, a+b, c), & \pm(b,-a-b, a+b+c), & \pm(a+2 b+2 c,-a-b-2 c, c), \\
\pm(-a-b, a, b+c), & \pm(a+b, b+2 c,-b-c), & \pm(a+b+c,-b-2 c, b+c), \\
\pm(-b,-a, a+b+c), & \pm(b, a+b+2 c,-a-b-c), & \pm(b+2 c,-a-b-2 c, a+b+c), \\
\pm(a+b,-b, b+c), & \pm(-a-b-2 c, a, b+c), & \pm(-a-b, a+2 b+2 c,-b-c), \\
\pm(-a, a+b+2 c,-c), & \pm(-b-2 c,-a, a+b+c), & \pm(-b, a+2 b+2 c,-a-b-c), \\
\pm(a, b+2 c,-c), & \pm(-a-2 b-2 c, a+b, c), & \pm(b+2 c,-a-2 b-2 c, a+b+c), \\
\pm(a+2 b+2 c,-b,-c), & \pm(a+b+2 c, b,-b-c), & \pm(-a-b-2 c, a+2 b+2 c,-b-c) .
\end{array}
$$

\section{References}

1. Billera, L.J., Gelfand, I.M., Sturmfels, B.: Duality and minors of secondary polyhedra. J. of Comb. Theory, Series B 57(2), 258-268 (1993)

2. Bodner, M., Patera, J., Szajewska, M.: Polytope contractions within icosahedral symmetry. Can. J. Phys. 92(11), 1446-1452 (2014). doi:10.1139/cjp-2014-0035

3. Chadzitaskos, G., Patera, J., Szajewska, M.: Polytopes vibrations within a reflection group symmetry submitted to EPJB (2015)

4. Champagne, B., Kjiri, M., Patera, J., Sharp, R.T.: Description of reflection generated polytopes using decorated Coxeter diagrams. Can. J. Phys. 73, 566-584 (1995)

5. Gilmore, R.: Lie Groups, Lie Algebras, and Some of Their Applications. Dover Publications (2006)

6. Humphreys, J.E.: Reflection Groups and Coxeter Groups. Cambridge University Press, Cambridge (1990)

7. Inönü, E., Wigner, E.P.: On the contraction of groups and their representations. Proc. Nat. Acad. Sci. 39(6), 510-524 (1953)

8. de Montigny, M., Patera, J.: Discrete and continuous graded contractions of Lie algebras and superalgebras. J. Phys. A Math. Gen. 24, 525-547 (1991)

9. Nguyen, X.: Posterior contraction of the population polytope in finite admixture models. Bernoulli 21(1), 618-646 (2015)

10. Ramond, P.: Group Theory: A Physicist's Survey. Cambridge University Press (2011) 